\title{
The Concept of "Risk Content" and its Calculation for the Investment Project Work
}

\author{
Margarita Doroshenko* \\ Vladimir State University, Institute of Tourism and Entrepreneurship, 87 Gorky str., Vladimir, \\ 600026, Russia
}

\begin{abstract}
The subject of the article is the concept of "risk content" introduced by the author and the peculiarities of its calculation for the investment project work. The purpose of the article is to provide an investor with practical tools for calculating the risk content of the project work. As a result of calculations, formulas for calculating resource losses and the risk content of the project work are found. The area of possible practical application of the research results is the investor's activity when investing in an investment project.
\end{abstract}

In developed countries with market economies, there are significant theoretical advances and practical experience in the field of economic risk theory, that is riskology, as evidenced by numerous translated publications that have recently appeared in our country. Most of these studies are devoted to the issues of risk in banking, financial and economic activities.

The term "risk" appeared in the works of Adam Smith, French researchers Jean-Gustave Courcelle-Seneuil and Jean-Baptiste Say. Later this category was deeply considered by Frank Hyneman Knight, J. R. Hicks, J. von Neumann, O. Morgenstern. Most of the papers of Russian scientists, such as I. Ansoff, I. T. Balabanov, V. V. Vitlinskiy, P. G. Graboviy, A. A. Pervozvansky, T. N. Pervozvanskaya, B. A. Reisberg V. T. Sevruk were limited to the study of the nature of risks, identifying their causes and classification, analysis and evaluation. However, a number of problems that are typical for making investment decisions in the modern Russian economy, related to the necessity to take into account risk, require further research.

Investments of private capital (both domestic and foreign) in capital construction, which, according to economic analysts, can become one of the ways to bailout Russian economy, are near zero due to the high level of associated risks, accounting of which is poorly formalized from the perspective of practicing investors.

The problem of determining the risk content of specific works of an investment project has not been given enough attention yet, and this tool is urgently needed for maximum forecasting of the risk situation associated with the resources loss, its foresight and taking preventive measures by the investor.

Andrew Flight was about to solve this problem in his book "Introduction to Project Finance" [1], where he defined the risks of individual project works: the risk of preparatory work, the risk of choosing a site and winning approval, the risks of performing work, etc.

*Corresponding author: doroshenrit@mail.ru 
Bykova R. G. in her article "Specific Nature of Risk Management in Project Activities" [2] considers the main actions for risk management at each stage of the project. Garkavaya L. in her paper "Project Risks: an Example, Risk Assessment, Analysis of Possible Events" [3] offers to carry out case analysis, analyze the situation in the industry and develop project implementation scenarios.

The purpose of the article is to introduce the concept of "risk content" of the investment project work and to give an investor practical tools for its calculation.

In accordance with the purpose of the work, the tasks are articulated as follows:

- $\quad$ to develop the concept of "risk content" of the investment project work;

- to identify groups of resources related to the project work;

- to introduce formulas for calculating resources loss;

- $\quad$ to develop formulas for calculating financial risk content of the work;

- to develop formulas for calculating risk content of the work as well as risk content of the work defined in compliance with the previously and later executed works;

- to give an example of calculating the risk content of the work of an investment project.

Risk-bearing factor (risk content) is a characteristic inherent in a work, event, procedure, document, or other conditional formalized medium that has adequate resources (financial, material, human, and moral) that can be lost partially or completely by an investor under certain conditions, called risk.

Investment is an integrated process involving various resources, which increase with the implementation of further works and stages of the investment cycle; similar idea is introduced in [4].

Each operation of the network schedule is characterized by a number of parameters. They are:

- name;

- duration;

- material resources involved in the work;

- human resources;

- financial resources, etc.

Certain work, as an elementary unit of the process as well as the network schedule, is associated with the movement of resources. Implementation of a particular work directly or indirectly affects the movement of resources. It is advisable to divide the resources related to the work into three conditional groups:

1 st group $-r_{i}$ includes resources directly involved in the process of performing this $i$-th work.

2nd group $-R^{B}$ involves resources related to the work performed earlier, before the i-th work, related to it by organizational and technological ties.

3rd group $-R^{L}$ are resources related to the work that will be performed later than the $\mathrm{i}$-th work, related to it by organizational and technological ties.

The concept of "risk content", introduced in this article, characterizes potentially existing losses of resources at a particular stage of the investment process or as a result of nonperformance of some specific work.

Similarly, there are three loss groups:

Group 1 includes resources losses $R_{i}$. They occur if the i-th work is not implemented and it is necessary to completely or partially redo it. Then you can write:

$$
R_{i}=r_{i} * k_{i}
$$

where $r_{i}$ is a resource for the $\mathrm{i}$-th work;

$k_{i}$ is the multiplicity of alterations of the i-th work. 
Group 2 are resources losses $R_{i}^{B}$, They occur as a result of non-performance of the i-th work, causing:

a) re-operation of all works performed before the i-th work, including the i-th; then the investment project continues;

b) cancelation of the investment project and irretrievable loss of all invested resources. It can be written as:

$$
R_{i}^{B}=\sum R_{i-n}
$$

where $R_{i-n}$ are resources for works performed before the i-th work, and at $0 \leqslant \mathrm{n}<\mathrm{i}$ they are equal to:

$$
R_{i-n}=r_{i-n} * k_{i-n},
$$

where $r_{i-n}$ are resources for each work completed before the i-th work;

$k_{i-n}$ is the multiplicity of work alterations up to the i-th work.

Group 3. Possible losses of resources $R_{i}^{L}$ for work that will be performed after the i-th work, and have organizational and technological connection with it.

$$
R_{i}^{L}=\sum R_{i+n},
$$

where $R_{i+n}$ includes the resources assigned to the works performed after the $\mathrm{i}$-th work, where $\mathrm{n}$ ranges from 0 to $\mathrm{N}$, where $\mathrm{N}$ is the number of project works.

$$
R_{i+n}=r_{i+n} * k_{i+n},
$$

where $r_{i+n}$ are resources for each work performed after the i-th work, but related to it organizationally and technologically;

$k_{i+n}$ is the multiplicity of alterations for each work after the i-th, related to it organizationally and technologically.

Thus, for each i-th work, three types of risk content are defined, or in other words, risk factors are defined:

$R_{i}$ is risk content of the $\mathrm{i}$-th work;

$R_{i}^{B}$ is risk content of the $\mathrm{i}$-th work in compliance with the previously performed work;

$R_{i}^{L}$ is the risk content of the $\mathrm{i}$-th work in compliance with the work completed later.

Considering investments as a single process, with a model in the form of a network graph, it is possible to distinguish the features of determining each type of risk content, namely:

$R_{i}$ - accumulates various types of resources, such as:

$M_{R i}$ - material resources;

$L_{R i}$ - human resources;

$S_{R i}$ - financial, etc.

In total, according to [5], there are 5 types of resources, namely: capital, people, materials, technologies, and information.

In this paper, we mainly consider financial resources, since they are the most important for the investor, in terms of possible risk. The financial risk content of the first work is a numerical, cost-based characteristic of it, i.e.

$$
\begin{gathered}
S_{P L i}=S_{R i}(P L) \\
\text { and } S_{I M P L i}=S_{R i}(I M P L) ;
\end{gathered}
$$

where $S_{I M P L i}$ is the planned capital investment for the implementation of the i-th work;

$S_{R i}(P L)$ is expected risk content of the first work;

$S_{I M P L i}$ is implemented capital investments in the performance of the i-th work;

$S_{R i}(I M P L)$ is actual risk content of the first work; 
The approach to defining $R_{i}^{B}$ and $R_{i}^{L}$ is similar. Due to the fact that all the work is connected by a network schedule in a single system, the implementation of each i-th work makes it possible to perform the next one.

$R_{i}^{B}$ is equal to the sum of all $R_{i}$ previous works without exception, i.e.

$$
R_{i}^{B}=\sum R_{i-n}
$$

For $R_{i}^{L}$ the situation is somewhat different. 2 judgments are valid here:

$R_{i}^{L}$ is equal to the sum of all $R_{i}$ following works without exception, i.e.

$$
R_{i}^{L}=\sum R_{i+n}
$$

Either $R_{i}^{L}$ is equal to the sum of all $R_{i}$ the works related to the $\mathrm{i}$-th organizational and technical relations; we will highlight them with the symbol $R_{i}^{L T R}$.

As we can see from the above, logically and mathematically, $R_{i}^{B}$ and $R_{i}^{L}$ are defined relatively simply. For each specific investment project, the investor needs to perform risk content calculations for each work at the planning stage. This will allow us to determine the necessary reserve funds to cover the risk of incorrect performance of the main, from a technological point of view, works. Methods for calculating resources for work are traditionally contained in the package of estimated financial calculations for projects (estimates, statements of materials and structures, construction arrangement project).

For the study of this topic, the introduction of the concept of risk content of works related to organizational and technological ties is of great importance, as one of the criteria for evaluating an investment project.

Example of calculating the risk content for work 1/2-1/3:

$$
R_{1 / 2-1 / 3}^{L T R}=R_{1 / 2-1 / 3}^{L T R}+R_{3 / 7-3 / 8 / 1}^{L T R}+R_{3 / 3-3 / 4 / 2}^{L T R}+R_{7 / 2-7 / 3 / 1}^{L T R}
$$

Work $1 / 2-1 / 3$ is the consideration of the customer's application letter for the approval of the construction site. In case the recommendations of the land management committee on this issue, which will be included in the draft approval letter, are interpreted and implemented incorrectly by the customer, later the project may not be agreed (work $3 / 7$ $3 / 8 / 1$ ) and the economy committee will express negative views regarding to the approval of the location of the object (work 3/3-3/4/2). And there may also be problems when approving the land acquisition project (work 7/2-7/3/1). The later the committee discovers the customer's mistakes, the more work has to be completely or partially redone, and the greater the losses the customer will suffer. Therefore, considering the risk content of work $1 / 2-1 / 3$, it is necessary to take into account not only the cost of resources required for its direct execution, but also the cost of resources of those works that are in organizational and technological connection with the original one, and these are works 3/7-3/8/1, 3/3-3/4/2 and $7 / 2-7 / 3 / 1$.

The investor should take into account the fact that the investment cycle may be terminated for whatever reason for any of the works. It is possible that a combination of unfavorable conditions and factors will make it unacceptable for the investor to continue implementing the planned program. To maximize the prediction of such a situation, its foresight and the adoption of proactive measures, the investor needs a tool. The network graph, as a model for determining the risk content, makes it possible to calculate optional scenarios in a real environment.

Thus, we can conclude that the purpose and objectives of the article are reached, namely, the concept of "risk content" of works of the investment project is developed; dedicated groups of resources related to the project are determined; calculation formula of resources losses are introduced; the formula for calculating financial "risk content" of works is provided; formula for calculating financial "risk content" of works as well as "risk content" 
of works regarding to the previously and later executed works are developed; there is an example of calculation of "risk content" of work of the investment project.

The author sees the prospects for further development of this direction in the formulation and calculation of the risk content of the investment project stages based on the risk content of the project works.

\section{References}

1. E. Flight, Introduction to project finance. 208, (2008)

2. R. G. Bykova, Bulletin of Omsk University, Specifics of Risk Management in Project Activities 4, 113-118 (2013)

3. Garkavaya L. Project risks: example, risk assessment, analysis of possible events [Electronic resource] - Access mode: FB.ru " article/445014/riski-proekta-primerotsenka...(Accessed 06.02.2021).

4. Doroshenko M. N. Proceedings of the 7th International Conference Innovation Management, Entrepreneurship and Sustainability, Prague, Statistical approach to the determination of numerical values of risk-benefit ratio in investment processes, 151-161 (2019).

5. Pervozvansky A. A., Pervozvanskaya T. N. Financial market: Calculation and Risk. 616 (1975). 